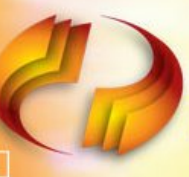

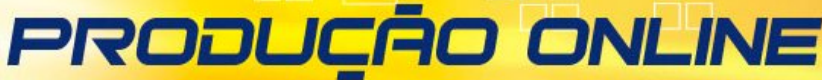

\section{A INFLUÊNCIA DA ENGENHARIA DE PRODUÇÃO NOS SERVIÇOS DE ATENDIMENTO À SAÚDE: ESTUDO BIBLIOMÉTRICO FOCADO EM TÉCNICAS OPERACIONAIS}

\section{THE INFLUENCE OF PRODUCTION ENGINEERING IN HEALTH CARE SERVICES: A BIBLIOMETRIC STUDY FOCUSED ON OPERATIONAL TECHNIQUES}

\author{
Maria Cecília Nascimento Rodrigues* E-mail: mcnrodrigues@sga.pucminas.br \\ Matheus Nunes de Lima Providello *E-mail: matheus.providello@sga.pucminas.br \\ Raoni Barros Bagno* E-mail: rbbagno@gmail.com \\ *Pontifícia Universidade Católica de Minas Gerais (PUC/MG), Belo Horizonte, MG
}

\begin{abstract}
Resumo: A Engenharia de Produção tem sua origem em aplicações essencialmente industriais, característica que acompanha a maior parte das técnicas e métodos abarcados por esta grande área de conhecimento ao longo do século XX. Entretanto, a reinterpretação e adequação deste conhecimento a contextos de produção essencialmente não industriais é um campo de vastas possibilidades. O presente trabalho toma as operações de atendimento à saúde como contexto de aplicação de técnicas de Engenharia de Produção e realiza um estudo bibliométrico em documentos indexados pela base de dados Scopus no período 2004-2014. Os dados obtidos foram organizados segundo várias dimensões como: autores, países, instituições e datas de publicação, evidenciando particularidades relativas a cada dimensão. Ao final, evidenciam-se oportunidades, tanto para a prática das unidades de atendimento quanto para a produção científica em Engenharia de Produção.
\end{abstract}

Palavras-chave: Engenharia de Produção. Hospital. Atendimento à saúde. Métodos e Técnicas Operacionais da Produção. Bibliometria.

\begin{abstract}
Production Engineering is originated on industrial applications, thereof developed methods and techniques along the last century were influenced by them. However, to the extent that such techniques spillover to non-industrial sectors, interesting opportunities arise. This paper presents a bibliometric study regarding the application of operational techniques that come from Production Engineering areas of study on health care services. The study is based on articles indexed by Scopus database from 2004 to 2014. Data collected was organized in several dimensions such as authors, countries, institutions and publication years and the main particularities were emphasized and discussed. Finally, some opportunities are identified both for the practice in health care units and new research in Production Engineering.
\end{abstract}

Keywords: Production Engineering. Hospital. Health care services. Bibliometry. Operational Methods and Production Techniques.

\section{INTRODUÇÃO}

Nos EUA, entre 1882 e 1912, foram registradas aplicações de técnicas e métodos do trabalho industrial que influenciaram o resto do mundo e até hoje continuam provocando transformações nas empresas. Como observado por Batalha Revista Produção Online, Florianópolis, SC, v. 16, n. 1, p. 242-262, jan./mar. 2016. 
(2008), o período Fordista/Taylorista está entrelaçado com o nascimento da Engenharia de Produção:

\begin{abstract}
A Engenharia de Produção começou, há mais de um século, com uma concepção de racionalidade econômica aplicada aos sistemas de produção. Coube a duas figuras paradigmáticas do final do século XIX e início do século XX o início da transformação dos conhecimentos empíricos sobre a produção em conhecimentos formalmente estabelecidos. Trata-se de Frederick Winslow Taylor e Henry Ford. (BATALHA, 2008)
\end{abstract}

A Indústria Bélica e a Indústria Automotiva constituem dois ramos de particular destaque como celeiro de métodos e técnicas que vieram a ser agregados às áreas de atuação da Engenharia de Produção. No exemplo de Ford, temos o pioneirismo dos automóveis em serem produzidos com partes padronizadas e intercambiáveis. Para isso, contudo, ele havia baseado em conceitos de engenharia desenvolvidos pelo exército americano na produção de armas, por volta de 1820 (BATALHA, 2008). Posteriormente, houve um importante marco no surgimento do Sistema Toyota de Produção, concebido a partir dos anos 1950 (SHINGO, 1996; OHNO, 1997) e cujos princípios e técnicas (como o Kanban e o Just-in-time) transbordaram a outras empresas e setores da indústria, proporcionando grandes saltos de produtividade (SILVA; SANTOS, 2001).

A Engenharia de Produção, como é conhecida no Brasil, já foi e ainda é conhecida como Engenharia Industrial em muitos países. De fato, uma grande parte das técnicas e métodos hoje atribuídos a áreas de estudo abarcadas pela Engenharia de Produção são oriundas da Indústria (FAÉ; RIBEIRO, 2005). Paulatinamente, conforme a adequação e contexto de cada sistema produtivo, observa-se um gradual transbordamento a outros setores industriais e também para serviços.

No contexto brasileiro, observou-se entre o início da década de 1990 e final dos anos 2000 um forte crescimento da oferta de vagas em cursos de Engenharia de Produção (BITTENCOURT; VIALI; BELTRAME, 2010). Tal difusão do conhecimento desta área pode ser visto como um catalisador, não somente da difusão das técnicas e conceitos abrangidos por suas áreas de conhecimento, como também de sua disseminação a contextos de produção diversificados. Um importante exemplo de tal diversificação está nas operações de saúde. A implantação de iniciativas como o caso do Lean Healthcare (BERTANI, 2012) são Revista Produção Online, Florianópolis, SC, v. 16, n. 1, p. 242-262, jan./mar. 2016. 
cada vez mais frequentes na agenda de trabalho das organizações. Outros exemplos são o Lean Office (SERAPHIM; DA SILVA; AGOSTINHO, 2010; EVANGELISTA; GROSSI; BAGNO, 2013) e também o Lean Service (FORTES, 2010; SOUSA; SILVEIRA; BAGNO, 2012).

A área da saúde tem crescente demanda por melhoria do desempenho de suas operações, mesmo em relação a outras áreas, já que acarreta em forte impacto social. Um exemplo pode ser dado na espera média por uma internação que, em 2004, estava na casa dos 4,5 dias (IPEA, 2006). Tal demanda representa uma oportunidade e, em certa medida, uma propensão para a adaptação e adoção com sucesso de técnicas e métodos de engenharia em áreas essencialmente não industriais. Assim como organizações produtivas de manufatura, unidades operativas de saúde (como hospitais) buscam cada vez mais se adequar a restrições de orçamentos, sem perda de qualidade nos serviços prestados variáveis clássicas envolvidas nos cálculos de eficiência produtiva. A dimensão geral do esforço pode ser obtida pelo número de instituições. No Brasil existem atualmente 6.617 hospitais sendo $21 \%$ municipais, $8 \%$ estaduais, $1 \%$ federais e $70 \%$ privados (CNS, 2014).

Este breve cenário ratifica a importância de se discutirem novos conceitos e técnicas que possam agregar produtividade às operações de saúde. No caso particular deste estudo, destaca-se a importância de se compreender como o aprendizado gerado nas aplicações clássicas de Engenharia de Produção pode e tem sido transbordado para as operações de saúde. Portanto, a questão central que permeia este estudo é: como as técnicas e ferramentas operacionais da Engenharia de Produção têm sido aplicadas na área de saúde em nível mundial, tomando como referência os registros extraíveis de bases de dados acadêmicas? Para isso, o trabalho recorre aos fundamentos da bibliometria para gerar os dados a serem analisados. Por "operacionais", entendam-se técnicas e ferramentas aplicáveis diretamente aos fluxos e processos fundamentais de transformação na produção, excluindo-se, assim, questões mais próximas do nível estratégico ou dos processos de suporte gerencial como sistemas de gestão, análise de mercado, gestão de pessoas ou sistemas de desenvolvimento de produtos e serviços, ainda que sejam reconhecidos seus desdobramentos aos níveis operacionais das organizações. 
Dentre os objetivos específicos desta pesquisa, procura-se identificar se existem centros de referência na reinterpretação e aplicação destas técnicas e métodos, se há autores que se destacam neste campo ou mesmo países que constituam escolas de destaque. Por fim, o estudo visa ainda identificar as lacunas e oportunidades para novos transbordamentos.

Devido à natureza particular deste estudo, as bases conceituais e metodológicas estão intimamente ligadas. Assim, na seção 2 são abordados os fundamentos da pesquisa bibliométrica e como se constituíram os passos adotados no estudo a partir destes fundamentos. A seção 3 apresenta os resultados obtidos no estudo em termos quantitativos e as suas respectivas análises, ao passo que a seção 4 ilustra algumas aplicações a partir da síntese de cinco artigos selecionados entre os resultados de busca. Por fim, na seção 5 é feita a conclusão acerca do estudo e dos resultados obtidos, suas principais contribuições, aplicações e também perspectivas para futuros trabalhos.

\section{BASE CONCEITUAL E METODOLOGIA}

\subsection{Cienciometria e Bibliometria}

De acordo com Crowl (1996), métodos quantitativos são usados para examinar questões que podem ser respondidas por meio de coleta e análise estatística de dados que se apresentam na forma numérica. Desta forma, a Cienciometria seria o estudo dos aspectos quantitativos da ciência enquanto uma disciplina ou atividade econômica. Trata-se de um segmento da sociologia da ciência, sendo aplicada no desenvolvimento de políticas científicas e envolve estudos quantitativos das atividades científicas, incluindo a publicação e, portanto, sobrepondo-se a Bibliometria (MACIAS-CHAPULA, 1998).

A origem da Cienciometria se deu na antiga URSS e na Europa Oriental e tem como ponto chave a atividade social, analisando a produção, o consumo e a circulação de publicações de cunho científico. Já a Bibliometria se configura como um recorte da Cienciometria e, conforme Macias-Chapula (1998), é o estudo dos aspectos quantitativos da produção, disseminação e uso da informação registrada. Assim, cabe à Bibliometria desenvolver padrões e modelos matemáticos para medir esses processos, usando seus resultados para elaborar previsões e apoiar tomadas

Revista Produção Online, Florianópolis, SC, v. 16, n. 1, p. 242-262, jan./mar. 2016. 
de decisões. A Cienciometria e a Bibliometria servem como mapa para mostrar a situação atual da produção científica e onde existem vácuos que possam ser preenchidos com a criação de produções científicas ou de onde os estudos podem usar como ponto de partida.

No Brasil, os estudos bibliométricos proliferaram na década de 1970, principalmente com os estudos realizados no então Instituto Brasileiro de Bibliografia e Documentação - IBBD (ARAÚJO, 2007). Como exemplo de estudos de cienciometria e bibliometria utilizados no ramo da Engenharia de Produção no Brasil podem-se citar os trabalhos de Andrade (2012), Lopes et al. (2012), Valle, Gutierrez e Costa (2013), Borba Prá e Miguel (2013) e Ariente Neto et al. (2014). De acordo com Araújo (2007), o uso de dados bibliométricos como indicadores da produção científica é de grande importância no planejamento nacional das atividades de pesquisa científica ou para análise do desenvolvimento da pesquisa científica e tecnológica em determinada instituição. Há ainda aplicações voltadas à análise dos periódicos, produtividade de pesquisadores ou determinação do léxico básico de um campo de pesquisa.

\subsection{Escolha e Formatação dos Termos e Pesquisa}

Sendo o objetivo deste trabalho relacionado ao transbordamento de métodos e técnicas operacionais da Engenharia de Produção aplicados a unidades de atendimento à saúde, a busca de artigos para uma análise bibliométrica demandou o levantamento prévio de termos a serem utilizados nas bases de dados. Assim, um primeiro momento foi marcado pela escolha de termos que representassem os métodos e técnicas da Engenharia de Produção condizentes com o recorte de pesquisa e um segundo momento envolveu o levantamento de termos que melhor representassem unidades operacionais de saúde, segundo nomenclatura adotada em linguagem acadêmica de língua inglesa. Conforme relatado com maior detalhe mais adiante, estes conjuntos de termos seriam então combinados em expressões booleanas aplicadas em base de dados acadêmica para subsequente seleção de artigos para análise.

A escolha dos termos relacionados a métodos e técnicas de Engenharia de Produção se iniciou pelo levantamento das áreas e sub-áreas da Engenharia de Revista Produção Online, Florianópolis, SC, v. 16, n. 1, p. 242-262, jan./mar. 2016. 
Produção por meio da Associação Brasileira de Engenharia de Produção (ABEPRO, 2014), em Currículos diversos de Engenharia de Produção de diversas Instituições de Ensino do Brasil e nas Diretrizes do Exame Nacional de Desempenho de Estudantes (ENADE) para a área de Engenharia de Produção (INEP, 2014). Os termos levantados nestes documentos foram pré-selecionados pelos autores tendo em vista o recorte proposto para o trabalho. Em seguida, a lista resultante foi encaminhada e discutida com cinco docentes da área de Engenharia de Produção com pelo menos 5 anos de experiência na academia para se levantar sugestões de ajustes. Destas interações foram então selecionados os doze termos finais para a pesquisa: Manufatura Enxuta, Mapa de Fluxo de Valor, Kanban, Just in Time, Teoria das Restrições, Teoria das Filas, 5S, Troca de Matrizes em um minuto (SMED), Manutenção Produtiva Total, Estudos de Tempos e Movimentos, Projeto de Layout, Análise Ergonômica do Trabalho. Evidentemente, a lista resultante não esgota os métodos e técnicas abordados na Engenharia de Produção mesmo no campo de aplicações de nível operacional. Entretanto, para garantir uma quantidade de termos que viabilizasse a pesquisa e, posteriormente, a análise dos dados, optou-se por evitar a predominância de subáreas como ocorreria com a Pesquisa Operacional, na sua enorme diversidade de métodos e técnicas em constante atualização.

Destes termos, resultaram então as seguintes expressões parciais para a pesquisa nas bases de dados: (Lean Manufacturing); (Value Stream Mapping); (Kanban); (Just in time) or (Just-in-time); (Theory of Constraints); (Queuing Theory); (5S) or (Seiri); (Single Minute Exchange of Die); (Total Productive Maintenance); (Time and Motion Study) or (Motion and Time Study) or (Time-Motion and (Study)) or (Methods Engineering); (Layout Design) and (Functional) or (Process) or (Product) or (Cells) or (Line) or (Project); (Ergonomic Analysis of Work) or (Ergonomic Analysis of Job) or ((Ergonomic) and (Work Analysis) or (Job Analysis)). Nota-se que, em vários dos casos, foram utilizados termos complementares ou alternativos para se evitar o retorno de documentos não aderentes aos objetivos da pesquisa entre os resultados de busca, ou mesmo para se evitar com que documentos relevantes não deixassem de ser capturados. Como exemplo, ao termo "5S", que, sozinho retornava resultados extremante diversificados, foi acrescido o termo "Seiri" (o segundo "S", associado ao senso de utilização) para que as respostas estivessem contextualizadas ao signficado de $5 \mathrm{~S}$ na Engenharia de Produção. Outro exemplo está nas combinações 
possíveis do termo Ergonomia com outros para se formar palavras-chave típicas de trabalhos desta área.

Já para os termos relacionados à área da saúde, primeiramente houve consultas formais e informais a docentes de cursos de graduação em medicina e profissionais da área para se levantar possíveis termos que melhor representassem as unidades operacionais de atendimento à saúde. Tal pesquisa foi complementada com consulta a dois documentos de suporte: (i) Portaria 4279 que corresponde à definição dos termos relacionados à área da saúde (MINISTÉRIO DA SAÚDE, 2010); (ii) Descritores em Ciências da Saúde, da Biblioteca Virtual em Saúde (BVS, 2014). Os termos resultantes foram: Hospital; Primary Health Care; Secondary Care; Tertiary Healthcare. Entretanto, ao longo das buscas iniciais da pesquisa, foi identificado que Primary Health Care, Secondary Care e Tertiary Healthcare não retornavam uma quantidade significativa de documentos adicionais ao termo "Hospital" (em outras palavras, artigos que retornavam com um destes termos, mas não retornavam com o termo "Hospital" eram raros). Assim, o termo "Hospital" foi utilizado sem outras combinações para capturar aplicações concernentes a unidades operacionais de atenção à saúde em geral. Escolhidos os termos relacionados às técnicas de Engenharia de Produção e adotado "hospital" como termos único para descrever unidades de atendimento à saúde, foram então geradas as expressões booleanas completas.

É importante, ressaltar, no entanto, que a utilização do termo "Hospital" não é suficiente para limitar os artigos retornados a reflexões e aplicações realizadas internamente no ambiente da unidade de atendimento à saúde. Como pode ser observado, por exemplo, no estudo de Diaz, Hendey e Bivins (2005), ao utilizar o termo "Hospital" são englobados estudos que tangenciam operações adjacentes às unidades de atendimento à saúde, como é o caso do serviço de ambulâncias. Optou-se, contudo, por não imprimir um esforço para exclusão destes estudos entre os resultados, dada a sua forte ligação com a temática do atendimento à saúde. Já estudos que citam o atendimento à saúde como exemplo em seus resumos, mas são voltados a outras áreas de serviço ou manufatura foram detectados e exluídos manualmente.

As expressões utilizadas foram: ("Lean Manufacturing") and (Hospital); ("Value Stream Mapping") and (Hospital); (Kanban) and (Hospital); ("Just in time") or Revista Produção Online, Florianópolis, SC, v. 16, n. 1, p. 242-262, jan./mar. 2016. 
("Just-in-time") and (Hospital); ("Theory of Constraints") and (Hospital); ("Queuing Theory") and (Hospital); (5S) and (Seiri) and (Hospital); ("Single Minute Exchange of Die") and (Hospital); ("Total Productive Maintenance") and (Hospital); ("Time and Motion Study") or ("Motion and Time Study") or (("Time-Motion") and (Study)) or ("Methods Engineering") and (Hospital); ("Layout Design") and (Functional) or (Process) or (Product) or (Cells) or (Line) or (Project) and (Hospital); ("Ergonomic Analysis of Work") or ("Ergonomic Analysis of Job") or ((Ergonomic) and ("Work Analysis") or ("Job Analysis") and (Hospital)).

A discussão acerca da base de dados a ser usada para um estudo desta natureza deve ser preferencialmente pautada em estudos comparativos, como, por exemplo, Meho e Yang (2007), Bar-Ilan (2008) ou Falagas et al. (2008). A base de dados escolhida para o estudo foi o Scopus devido a sua maior abrangência e múltiplas possibilidades de análise dos resultados, como, por exemplo, países e instituições de origem dos trabalhos retornados. Foi considerado um período de dez anos para o levantamento de dados (2004 a 2014). Foram considerados como documentos válidos para a pesquisa artigos de períodicos, eventos, capítulos de livros, editoriais e relatórios.

Durante o levantamento de dados, dois termos se destacaram nos extremos quanto ao número de estudos retornados. A expressão relacionada aos Estudos de Tempos e Movimentos obteve um número de resultados muito acima das demais, na casa de 600 estudos. Por este motivo, decidiu-se realizar uma discussão em separado sobre os resultados associados a este termo. No outro extremo, a expressão relacionada à Troca de Matrizes em um minuto (SMED) retornou apenas 1 documento, o que motivou um detalhamento diferenciado deste resultado. Os dados dos documentos resutantes das demais expressões foram agrupados em tabelas segundo categorias: palavra-chave, autores, número de citações, data, instituições e países de origem. A próxima seção discute os principais pontos que emergiram dos agrupamentos realizados.

\section{ANÁLISE DOS DADOS E RESULTADOS}

A figura 1 evidencia o número de resultados no mecanismo de pesquisa para cada expressão de entrada, cujo total ficou em 1033 documentos. Primeiramente 
será analisado o Estudo de Tempos e Movimentos, que apresentou um retorno muito alto em comparação aos demais termos e, na sequência, o documento relacionado ao SMED. As análises sobre as demais expressões vem a seguir.

Quatro países respondem por 60\% dos documentos retornados: EUA (39\%), China (8,5\%), Canadá (6,5\%) e Reino Unido (6\%). Este quadro pouco difere da produção científica geral no mundo, em que estes mesmos países se encontram, respectivamente, nas $1^{\circ}, 2^{\circ} \underline{0}, 7^{\circ}$ e e $4^{\circ}$ posições, segundo estatísticas da Thomson Reuters. O Brasil também mantém sua posição relativa, respondendo por 2,3\% dos resultados da pesquisa ante $2,5 \%$ de sua contribuição geral no cenário da pesquisa mundial (FOLHA DE SÃO PAULO, 2014).

Figura 1 - Quantidade de Artigos x Palavra-chave

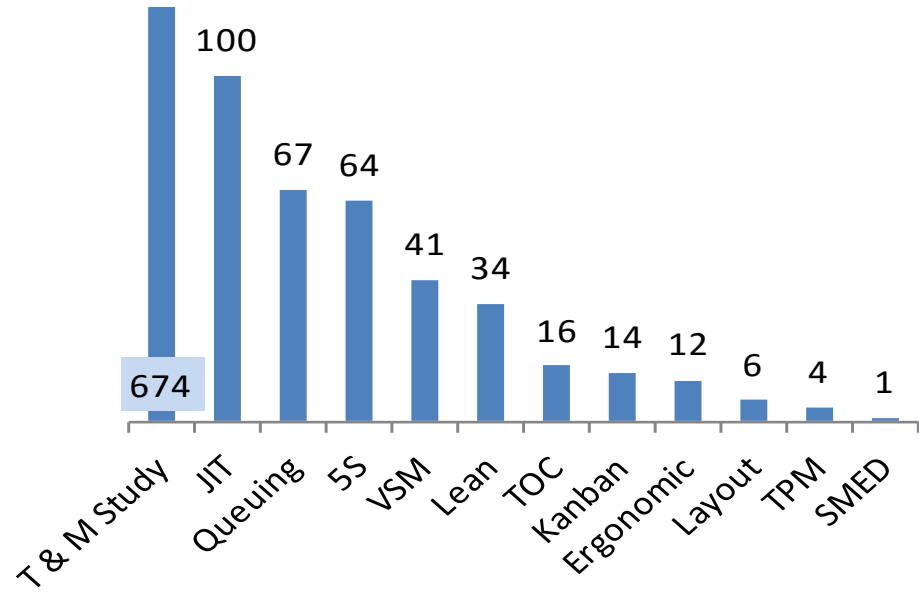

Fonte: Dos Autores

Aparte à quantidade de documentos relativos ao Estudo de Tempos e Movimentos é possível notar que os outros termos não apresentam variações expressivas na quantificação de seus resultados. Como exemplo, o desvio-padrão da quantidade de documentos retornados do JIT, Teoria das Filas, 5S, Mapa de Fluxo de Valor e Manufatura Enxuta é equivalente a 25,9 com uma média de 61,2 documentos.

\subsection{Estudo e Tempos e Movimentos e Troca de Matrizes em um Minuto}

O Estudo de Tempos e Movimentos retornou na pesquisa 674 documentos, o que representa $65,25 \%$ do total. Pode-se supor que a consolidação deste tipo de 
estudo ao longo de muitas décadas e a transversalidade a diversas situações de trabalho tenham influenciado este quadro. Não foi identificado, contudo nenhum autor ou instituição que se sobressaia sobre os demais de forma relevante. O autor que mais retornou nos resultados foi Westbrook (Universidade de New South Wales, Sydney, Australia) com 10 documentos (tabela 1). No entanto, no que tange ao rol de instituições representadas, as duas primeiras posições não estão reservadas a universidades: a VA Medical Center aparece em primeiro com 17 documentos que retornaram e em segundo aparece a Massachusetts General Hospital com 13 documentos (tabela 2).

Tabela 1 - Principais Autores e suas Instituições (Estudo de Tempos e Movimentos)

\begin{tabular}{l|c|c}
\hline \multicolumn{1}{c|}{ Autor } & Resultados & Instituição \\
\hline Westbrook, J.I. & 10 & University of New South Wales (UNSW) Australia, Sydney \\
\hline Groneberg, D.A. & 7 & Johann Wolfgang Goethe Universitat Frankfurt am Main \\
\hline Mache, S. & 7 & Johann Wolfgang Goethe Universitat Frankfurt am Main \\
\hline Bradley, E.H. & 6 & Yale University, Global Health Leadership Institute, New Haven, USA \\
\hline Klapp, B.F. & 6 & Charite - Universitats medizin Berlin, Department of Medicine/Psychosomatics, Berlin, Germany \\
\hline Sack, H. & 5 & Klinikum rechts der Isar, Department of Radiotherapy and Radiooncology, Munich, Germany \\
\hline Popp, W. & 5 & Klinikum rechts der Isar, Department of Radiotherapy and Radiooncology, Munich, Germany \\
\hline Krumholz, H.M. & 5 & Yale University, Global Health Leadership Institute, New Haven, USA \\
\hline Herrin, J. & 4 & Health Research and Educational Trust HRET, Chicago, United States \\
\hline
\end{tabular}

Fonte: Dos Autores

A VA Medical Center é uma instituição criada nos EUA para veteranos de guerra. Neste país existe um dos mais abrangentes sistemas de apoio aos veteranos de guerra e/ou deficientes provenientes da guerra. A VA Medical Center possui várias unidades em todo o país (USDVA, 2014). Já o Massachusetts General Hospital, que se encontra em Boston, EUA, é uma instituição de saúde criada por médicos e cientistas, na qual declara-se haver um forte relevante histórico e forte incentivo a novas pesquisas (MGH, 2014).

Tabela 2 - Principais Instituições (Estudo de Tempos e Movimentos)

\begin{tabular}{l|c}
\hline \multicolumn{1}{|c|}{ Instituição } & Resultados \\
\hline VA Medical Center & 17 \\
\hline Massachusetts General Hospital & 13 \\
\hline University of Sydney & 12 \\
\hline Brigham and Women's Hospital & 10 \\
\hline University of New South Wales UNSW Australia & 10 \\
\hline University of Toronto & 9 \\
\hline Yale University School of Medicine & 8 \\
\hline University of California, San Francisco & 8 \\
\hline Charite - Universitatsmedizin Berlin & 8 \\
\hline University of Melbourne & 8 \\
\hline
\end{tabular}

Fonte: Dos Autores

Revista Produção Online, Florianópolis, SC, v. 16, n. 1, p. 242-262, jan./mar. 2016. 
No outro extremo dos resultados encontrados com as expressões utilizadas na pesquisa está o Single Minute Exchange of Die (SMED), ou Troca de Matrizes em um minuto, que retornou apenas um documento. O artigo intitulado "Lean maintenance - A risk-based approach", de Clarke, Mulryan, e Liggan, foi publicado em 2010 no periódico Pharmaceutical Engineering. Este periódico é promovido pelo ISPE - Sociedade Internacional de Engenharia Farmacêutica, que ostenta o status de maior associação não-lucrativa do mundo nesta área (ISPE, 2015).

O SMED é uma técnica relacionada ao contexto da manufatura enxuta. No artigo em questão, pode-se observar que a abordagem não é somente sobre Troca de Matrizes em um minuto, e sim sobre técnicas relacionadas ao Toyotismo em geral. O texto discorre acerca da implantação da manutenção enxuta na Pfizer Biotech em Dublin, Irlanda. Dentre as conclusões, os autores afirmam ter obtido uma redução de $25 \%$ no tempo de inatividade de um bioreator, por meio da preparação e simplificação de tarefas (CLARKE; MULRYAN; LIGGAN, 2010).

\subsection{Demais Expressões}

A tabela 3 mostra o ranking dos documentos mais citados. Apesar de Teoria das Filas não ser a área em que retornou o maior número de estudos, três trabalhos a ela relacionados lideram a lista em número de citações. Entretanto, conforme pode ser visto na tabela 4, os autores mais produtivos se concentram mais nos temas do Mapa de Fluxo de Valor e Manufatura Enxuta. Em se tratando do número de citações, vale observar que textos mais antigos tendem a ser privilegiados em número de citações. Entretanto, somente o terceiro texto da tabela 3 corresponde ao ano de 2004, que marca o recorte inicial das buscas. 
Tabela 3 - Estudos mais Citados

\begin{tabular}{|c|c|c|c|c|c|}
\hline Palavra-Chave & Autor & Citado & Estudo & Publicado em & Ano \\
\hline $\begin{array}{l}\text { Queuing } \\
\text { Theory }\end{array}$ & Hoot, N.R., Aronsky, D. & 242 & $\begin{array}{l}\text { Systematic Review of Emergency Department } \\
\text { Crowding: Causes, Effects, and Solutions }\end{array}$ & $\begin{array}{l}\text { Annals of Emergency } \\
\text { Medicine }\end{array}$ & 2008 \\
\hline $\begin{array}{l}\text { Queuing } \\
\text { Theory }\end{array}$ & $\begin{array}{l}\text { King, D.L., Ben-Tovim, D.I., } \\
\text { Bassham, J. }\end{array}$ & 81 & $\begin{array}{l}\text { Redesigning emergency department patient } \\
\text { flows: Application of Lean Thinking to health } \\
\text { care }\end{array}$ & $\begin{array}{l}\text { EMA - Emergency Medicine } \\
\text { Australasia }\end{array}$ & 2006 \\
\hline $\begin{array}{l}\text { Queuing } \\
\text { Theory }\end{array}$ & $\begin{array}{l}\text { McManus, M.L., Long, M.C., } \\
\text { Cooper, A., Litvak, E. }\end{array}$ & 71 & $\begin{array}{c}\text { Queuing Theory Accurately Models the Need } \\
\text { for Critical Care Resources } \\
\end{array}$ & Anesthesiology & 2004 \\
\hline Lean & $\begin{array}{l}\text { Dickson, E.W., Singh, S., } \\
\text { Cheung, D.S., Wyatt, C.C., } \\
\text { Nugent, A.S. }\end{array}$ & 52 & $\begin{array}{l}\text { Application of Lean Manufacturing Techniques } \\
\text { in the Emergency Department }\end{array}$ & $\begin{array}{l}\text { Journal of Emergency } \\
\text { Medicine }\end{array}$ & 2009 \\
\hline $5 S$ & $\begin{array}{l}\text { Roca-Feltrer, A., Carneiro, I., } \\
\text { Armstrong Schellenberg, } \\
\text { J.R.M. }\end{array}$ & 40 & $\begin{array}{l}\text { Estimates of the burden of malaria morbidity } \\
\text { in Africa in children under the age of } 5 \text { years }\end{array}$ & $\begin{array}{l}\text { Tropical Medicine and } \\
\text { International Health }\end{array}$ & 2008 \\
\hline
\end{tabular}

Fonte: Dos Autores

Decidiu-se não optar por uma "diluição" das publicações em coautoria para os fins deste estudo, uma vez que o interesse está voltado para o envolvimento de pesquisadores e instituições com os temas em questão, e não o mérito científico propriamente dito. No exemplo, os autores brasileiros Calado e Coelho são parceiros em dois artigos publicados em 2013, de forma que ambos foram contabilizados para os dois autores. Outro ponto é que não raramente, tais documentos retornam em mais de uma expressão de busca, devido à afinidade conceitual existente entre eles, como evidenciado na tabela 4.

Tabela 4 - Autores mais Produtivos

\begin{tabular}{l|c|l|l}
\hline \multicolumn{1}{c|}{ Autor } & Estudos & \multicolumn{1}{|c}{ Tema } & \multicolumn{1}{|c}{ Instituição } \\
\hline Wang, S. & 6 & $\begin{array}{l}\text { Lean Manufacturing e Value } \\
\text { Stream Mapping }\end{array}$ & $\begin{array}{l}\text { University of Akron, Department of } \\
\text { Mechanical Engineering (USA) }\end{array}$ \\
\hline Calado, R. D. & 4 & $\begin{array}{l}\text { Lean Manufacturing e Value } \\
\text { Stream Mapping }\end{array}$ & $\begin{array}{l}\text { Universidade Sao Francisco, Industrial } \\
\text { Engineering Graduation Course, Itatiba, } \\
\text { Brazil }\end{array}$ \\
\hline Chadha, R. & 4 & $\begin{array}{l}\text { 5S, Just in Time, Queueing } \\
\text { Theory e Value Stream } \\
\text { Mapping }\end{array}$ & Mosaic Potash ULC, Saskatoon, Canada \\
\hline Coelho, S. M. & 4 & $\begin{array}{l}\text { Lean Manufacturing e Value } \\
\text { Stream Mapping }\end{array}$ & Instituto de Oncologia Do Vale, Brazil \\
\hline Hogg, W. & 4 & Just in Time & $\begin{array}{l}\text { University of Ottawa, Department of } \\
\text { Family Medicine, Canada }\end{array}$ \\
\hline
\end{tabular}

Fonte: Dos Autores

No que tange às instituições de origem, a tabela 5 lista as cinco principais, em relação ao número de trabalhos. Pode-se observar também por estes dados que as contribuições são bastante pulverizadas - considerando um montante de mais de 1000 estudos publicados nos últimos dez anos, não há nenhuma institução que concentre uma dezena de contribuições. Chama a atenção, no entanto, a 
participação de membros das próprias instituições de saúde no desenvolvimento dos trabalhos, seja em parceria com universidades, seja conduzindo linhas de pesquisa próprias.

Tabela 5 - Principais Instituições

\begin{tabular}{l|c|c}
\hline \multicolumn{1}{c|}{ Instituição } & Estudos & Tipo de Instituição \\
\hline University Michigan Ann Arbor & 7 & Universidade \\
\hline Massachusetts General Hospital & 5 & Instituição de saúde \\
\hline Mayo Clinic & 5 & Instituição de saúde \\
\hline University of Ottawa, Canada & 5 & Universidade \\
\hline Children's Hospital and Regional Medical Center & 4 & Instituição de saúde \\
\hline
\end{tabular}

Fonte: Dos Autores

A figura 2 mostra os documentos retornados na linha do tempo. Apesar de não se poder identificar uma tendência constante de crescimento ou mesmo de queda na produção científica relacionada aos termos de estudo, a média anual de publicações dos seis últimos anos é significativamente maior do que os cinco primeiros anos do período pesquisado.

Figura 2 - Documentos x Data de Publicação

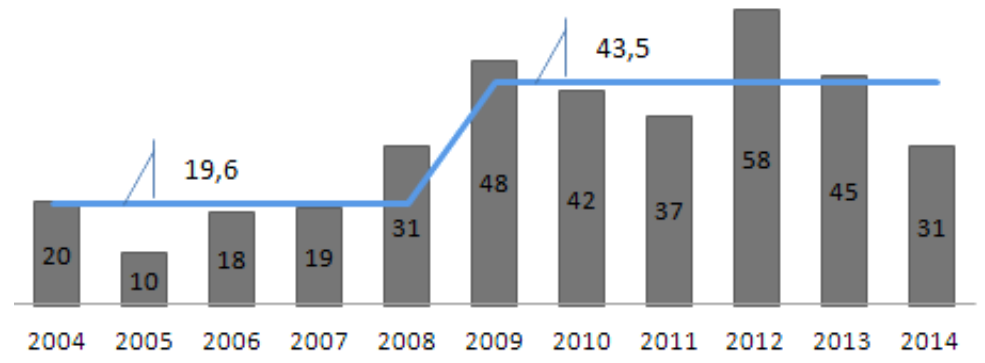

Fonte: Dos Autores

Em geral, pode-se afirmar que os números obtidos representam uma pequena fração do esforço de aplicação e reinterpretação de métodos e técnicas da Engenharia de Produção no campo da saúde. O fato de o estudo se concentrar em documentos retornados de uma base científica indexada mostra somente a parte deste esforço que resulta em publicações científicas de maior relevância, apesar de a busca ter sido relativamente inclusiva quanto aos tipos de documentos considerados, uma das vantagens da base Scopus para este propósito. De qualquer forma, os trabalhos obtidos nos resultados demonstram importantes exemplos de transbordamentos para a área de saúde, ao mesmo tempo em que os números 
mostram um vasto campo para novas aplicações. Em parte, isso é ratificado pelo destaque dos resultados associados aos Estudos de Tempos e Movimentos.

É importante destacar a importância das parcerias entre instituições acadêmicas e instiuições de saúde para a geração de resultados. Vários dos estudos retornados nas buscas evidenciam este tipo de parceria. Um interessante exemplo no Brasil, está no trabalho de Pinto et al. (2014), que aplicou as técnicas de simulação e teoria de filas para analisar a capacidade de leitos hospitalares. $O$ autores envolvidos no estudo representam três diferentes departamentos de uma universidade pública (Engenharia de Produção, Ciências Econômicas e Educação em Saúde Pública) e um hospital. Entre 349 artigos de 36 países, o trabalho foi selecionado como o melhor artigo na área de simulação aplicada, apresentado no Winter Simulation Conference de 2014 (WSC, 2014), congresso internacional de simulação de expressiva relevância. Este exemplo demonstra como, apesar da ainda tímida contribuição de estudos brasileiros que lidem com a aplicação de métodos e técnicas de engenharia na área da saúde, iniciativas locais apoiadas em parcerias podem alcançar grandes resultados, tanto para a prática das unidades de saúde, quanto para o desenvolvimento acadêmico-científico

\section{EXEMPLOS DE ESTUDOS SELECIONADOS}

O objetivo da presente seção é trazer alguns exemplos de estudos retornados na pesquisa com o objetivo de ilustrar a aplicações das técnicas selecionadas para o processo de busca na base de dados. Para este propósito, foram escolhidas cinco expressões: JIT, Teoria das Filas, 5S, Mapa de Fluxo de Valor e Manufatura Enxuta, que são as expressões que retornaram mais estudos após Estudo de Tempos e Movimentos. Dos resultados destas expressões, foram selecionados documentos entre os mais citados para compor os comentários desta seção. Como os documentos podem se localizar nas intercessões entre os resultados de busca de cada expressão, optou-se por não associar diretamente cada subseção a uma respectiva palavra-chave. Seguem abaixo os estudos com suas respectivas sínteses:

- Hoot e Aronsky (2008): Revisão sistemática de aglomerações no departamento de emergência: causas, efeitos e soluções. O estudo citado Revista Produção Online, Florianópolis, SC, v. 16, n. 1, p. 242-262, jan./mar. 2016. 
foi desenvolvido por autores da Vanderbilt University Medical Center (Tennessee, EUA), sendo o primeiro do Departamento de Informática Biomédica e o segundo do Departamento de Medicina de Emergência. $O$ texto retoma a literatura existente sobre superlotação em unidades de saúde. Durante o trabalho de revisão os autores selecionam 93 artigos: 33 que estudam as causas, 27 que estudam os efeitos e 40 que estudam as soluções para a superlotação das unidades de saúde. A conclusão do trabalho destaca a recente importância da utilização de técnicas de engenharia em Unidades de Saúde, realizando uma comparação com outros setores como o de aviação e manufatura nos quais há maior maturidade de aplicação destas técnicas. Como exemplo, é citado o uso de Teoria das Filas em aplicações voltadas a melhorar o fluxo de pacientes em Unidades de Saúde.

- Diaz, Hendey e Bivins (2005): Quando é o helicóptero mais rápido? Uma comparação dos tempos de transporte de helicóptero e ambulância terrestre. Os autores são do Departamento de Medicina de Emergência do St. Mary Medical Center (Califórnia, EUA). A questão central de pesquisa está diretamente associada ao título do trabalho e a análise baseia-se nos tempos de 7854 ambulâncias e 1075 helicópteros. Foram criados três grupos para análise: terreno, helicóptero enviado juntamente com a unidade terrestre e helicóptero não enviado simultaneamente. $\mathrm{O}$ estudo apontou ser a ambulância terrestre mais adequada quando a distância é inferior a 10 milhas do hospital. O transbordamento do estudo para Unidades de Saúde diversas pode não somente melhorar a qualidade de atendimento, mas também reduzir custos.

- Ng et al. (2010): Aplicação dos princípios enxutos do sistema Toyota de produção para reduzir tempos de espera no departamento de emergência. Os autores são do Departamento de Emergência do Hospital Hôtel-Dieu Grace (Ontario, Canadá). Em 2015, o Hospital Hôtel-Dieu Grace começou um processo de implantação dos Princípios da Manufatura Enxuta, incluindo técnicas como o Estudo de Tempos e Movimentos, Mapeamento do Fluxo de Valor e Just-in-time. Após o período de implantação, a unidade obteve melhorias como no tempo Revista Produção Online, Florianópolis, SC, v. 16, n. 1, p. 242-262, jan./mar. 2016. 
médio de consulta, que caíra de 111 minutos para 78 e no número pacientes que saem sem ser atendidos, que passara de 7,1\% para 4,3\%. Outras variáveis afetadas foram o tempo da duração da estadia dos pacientes o nível de satisfação observado pelos clientes em relação aos serviços ofertados.

- Dickson et al. (2009): Aplicação de técnicas de manufatura enxuta no departamento de emergência. A maior parte dos autores é ligada ao departamento de medicina de emergência da Universidade de lowa (EUA). O estudo relata a aplicação técnicas de Manufatura Enxuta em uma Unidade de Saúde como forma de tentar melhorar a satisfação de pacientes e equipe, pontos em que são relatadas melhorias após a implantação das práticas. Dentre as principais conclusões, relata-se que a tentativa de aplicar as técnicas revoga a prática de cópia das ações de mudanças de processos utilizadas em outras instituições em favor da observação dos princípios fundamentais da Manufatura Enxuta, com a devida contextualização ao ambiente real de uso.

- Rutledge, Xu e Simpson (2010): Aplicação do sistema Toyota de produção melhora as operações centrais de laboratório. Os autores são do Departamento de Laboratórios do Hospital Infantil de Seattle, da Escola de Medicina da Universidade de Washington (EUA). As abordagens utilizadas neste estudo incluem o 5S, trabalho padronizado e fluxo de peça única. As variáveis impactadas foram o tempo de resposta de um dos testes realizados, reduzido de 54 minutos para 23 e também o volume de testes realizados, que aumentou em $20 \%$. São ainda citados economia monetária, diminuição de variabilidade de tempo de execução de testes e melhor utilização do espaço físico. O texto recomenda ainda o uso do método PDCA durante os quatro anos subsequentes como forma de manutenção e continuidade das melhorias.

Certamente o universo de estudos levantados não permite uma análise detalhada das possibilidades de aplicação das técnicas operacionais de Engenharia de Produção na saúde. Contudo, os exemplos dados destacam pontos em comum no uso de princípios de produtividade (como análise e medição, quantificação das Revista Produção Online, Florianópolis, SC, v. 16, n. 1, p. 242-262, jan./mar. 2016. 
variáveis em estudo), abordagens de gestão para manutenção dos resultados e transbordamentos das aplicações realizadas para o ambiente social das instituições.

\section{CONSIDERAÇÕES FINAIS}

Este trabalho teve o intuito de fomentar a discussão acerca de possíveis transbordamentos da Engenharia de Produção na área da saúde, focando-se em um conjunto pré-selecionado de métodos e técnicas de maior influência no ambiente operacional. Deve-se reconhecer a limitação intrínseca causada pelo recorte de pesquisa, que opta por alguns termos em detrimento de outros. No entanto, o estudo evidencia possibilidades, avanços e oportunidades para aplicação de esforços com vistas a resultados tangíveis no atendimento à saúde e também a contribuições de cunho científico. Para a Engenharia de Produção, enquanto área de atuação profissional, este estudo evidencia também grandes oportunidades fora do meio essencialmente industrial, que se encontra na raiz da área de conhecimento e de praticamente todos os métodos e técnicas nela desenvolvidos.

O uso da bibliometria como forma de medição deste transbordamento reservou alguns desafios quanto à complexidade da informação gerada e das próprias formas de montagem das expressões a serem aplicadas no mecanismo de busca, mas demonstrou ser uma importante alternativa para compreensão da penetração dos estudos de uma área (métodos e técnicas de nível operacional da Engenharia de Produção) em um determinado contexto de aplicação (unidades de atendimento de saúde). As etapas deste estudo podem ser reinterpretadas e reproduzidas para outras áreas de conhecimento e contextos, como forma de evidenciar avanços e oportunidades.

Os dados gerados para este estudo possibilitam aprofundamentos detalhados para cada método ou técnica considerado ou mesmo sua extrapolação para recortes nos níveis tático e estratégico, não considerados para o presente trabalho. Deve-se considerar ainda que a produção científca é dinâmica, o que demanda constante atualização dos dados. É importante observar que muitos dos métodos e técnicas considerados na pesquisa representam, para o meio industrial, exemplos do estado das práticas e são pouco considerados em novas pesquisas de cunho científico. No 
entanto, sua reinterpretação e aplicação em outros contextos em que se enquadrem os sistemas produtivos ainda podem gerar grandes trasnformações.

Este esforço de pesquisa pode ser usado como ponto de partida para novas investigações com recortes mais particularizados e também para fomentar aplicações práticas. O nortemento para se buscar parcerias com instituições externas de destaque nos estudos levantados também surge como possível contribuição. Entretanto, em síntese, vale destacar o forte potencial do uso de métodos e técnicas de Engenharia de Produção - ainda que já consolidados em seu ambiente original de desenvolvimento - em contextos produtivos emergentes.

\section{REFERÊNCIAS}

ABEPRO. Áreas e Sub-áreas de Engenharia de Produção. 2014. Disponível em: < http://www.abepro.org.br/interna.asp? $\mathrm{p}=399 \& \mathrm{~m}=424 \& s s=1 \& \mathrm{c}=362$ >. Acesso em: $10 \mathrm{Jul}$ 2014

ANDRADE, F.S. Análise Bibliométrica da Produção Científica de Pesquisadores e Referências de um Periódico da Engenharia de Produção. 2012. 75 f. (Mestrado profissional). Escola de Engenharia do Rio Grande do Sul, Universidade Federal do Rio Grande do Sul - UFRGS, Porto Alegre.

ARAÚJO, C.A. Bibliometria: evolução histórica e questões atuais. Em Questão, v. 12, n. 1, 2007.

ARIENTE NETO, R.; SILVA, S.L.; FORCELLINI, F.; FERREIRA, M.G.G.; MIGUEL, P.A.C. Elementos integrantes de um sistema produto-serviço como potencial para alcançar um desenvolvimento sustentável: bibliometria e análise de conteúdo. Revista Produção Online, v. 14, n. 3, p. 914-938, 2014. http://dx.doi.org/10.14488/1676-1901.v14i3.1566

BAR-ILAN, J. Which h-index?-A comparison of WoS, Scopus and Google Scholar. Scientometrics, v. 74, n. 2, p. 257-271, 2008. http://dx.doi.org/10.1007/s11192-008-0216-y

BATALHA, M.O. Introdução à engenharia de produção. Rio de Janeiro: Elsevier, 2008.

BERTANI, T.M. Lean Healthcare: Recomendações para implantações dos conceitos de produção enxuta em ambientes hospitalares. 2012. $166 \mathrm{f}$ (Mestrado em Engenharia de Produção). Escola de Engenharia de São Carlos, Universidade de São Paulo, São Carlos.

BITTENCOURT, H.R.; VIALI, L.; BELTRAME, E. A engenharia de produção no Brasil: um panorama dos cursos de graduação e pós-graduação. Revista de Ensino de Engenharia, v. 29, n. 1, 2010.

BORBA PRÁ, F.; MIGUEL, P.A.C. Evolução na aplicação do QFD: análise de publicações qualificadas em periódicos. Exacta, v. 11, n. 1, p. 89-100, 2013.

BVS. Descritores em Ciências da Saúde. 2014. Disponível em: < http://decs.bvs.br/ >. Acesso em: 15 Ago 2014. 
CLARKE, G.; MULRYAN, G.; LIGGAN, P. Lean Maintenance-A Risk-Based Approach. Pharmaceutical Engineering, v. 30, n. 5, p. 1-6, 2010.

CNS. Dados do Setor. 2014. Disponível em: < http://www.cns.org.br/links/DADOS DO SETOR.htm >. Acesso em: 20 Dez 2014.

CROWL, T.K. Fundamentals of educational research. McGraw-Hill Humanities, Social Sciences \& World Languages, 1996.

DIAZ, M.A.; HENDEY, G.W.; BIVINS, H.G. When is the helicopter faster? A comparison of helicopter and ground ambulance transport times. Journal of Trauma and Acute Care Surgery, v. 58, n. 1, p. 148-153, 2005. http://dx.doi.org/10.1097/01.TA.0000124264.43941.41

DICKSON, E.W.; SINGH, S.; CHEUNG, D.S.; WYATT, C.C.; NUGENT, A.S. Application of Lean Manufacturing techniques in the emergency department. Journal of Emergency Medicine, v. 37, n. 2, p. 177-182, Aug 2009. http://dx.doi.org/10.1016/j.jemermed.2007.11.108

EVANGELISTA, C.S.; GROSSI, F.M.; BAGNO, R.B. Lean Office-escritório enxuto: estudo da aplicabilidade do conceito em uma empresa de transportes. Revista Eletrônica Produção \& Engenharia, v. 5, n. 1, p. 462-471, 2013.

FAÉ, C.S.; RIBEIRO, J.L.D. Um retrato da engenharia de produção no Brasil. Revista Gestão Industrial, v. 1, n. 03, p. 315-324, 2005. http://dx.doi.org/10.3895/S1808$\underline{04482005000300003}$

FALAGAS, M.E.; PITSOUNI, E.I.; MALIETZIS, G.A.; PAPPAS, G. Comparison of PubMed, Scopus, web of science, and Google scholar: strengths and weaknesses. The FASEB journal, v. 22, n. 2, p. 338-342, 2008. http://dx.doi.org/10.1096/fj.07-9492LSF

FOLHA DE SÃO PAULO. Em 20 anos, país vai de 240. a 130. em raking de pesquisa. 2014. Disponível em: < http://www1.folha.uol.com.br/ciencia/2014/11/1541834-em-20-anospais-vai-de-24-a-13-em-ranking-de-pesquisa.shtml >. Acesso em: 12 Jan 2015.

FORTES, C.S. Aplicabilidade de Lean Service na melhoria de serviços de Tecnologia da Informação (TI). 2010. 168 f. (Mestrado profissional em Engenharia de Produção). Escola de Engenharia do Rio Grande do Sul, Universidade Federal do Rio Grande do Sul UFRGS, Porto Alegre.

HOOT, N.R.; ARONSKY, D. Systematic review of emergency department crowding: causes, effects, and solutions. Annals of emergency medicine, v. 52, n. 2, p. 126-136. e1, 2008. INEP. Portaria Inep $n^{\circ}$ 249, de 02 de junho de 2014. 2014. Disponível em: < http://download.inep.gov.br/educacao superior/enade/legislacao/2014/diretrizes cursos dipl omas bacharel/diretrizes bacharel engenharia producao.pdf $>$. Acesso em 10 Fev 2014.

IPEA. Saúde-Tempo Real. 2006. Disponível em: < http://www.ipea.gov.br/desafios/index.php?option=com content\&view=article\&id=991:reporta gens-materias\&ltemid=39 >. Acesso em: 03 Fev 2015.

ISPE. International Society for Pharmaceutical Engineering - Institutional Web Site. 2015. Disponível em: < http://www.ispe.org >. Acesso em: 25 Mai 2015.

LOPES, A.P.; KISSIMOTO, K.O.; SALERNO, M.S.; LAURINDO, F.J.B.; CARVALHO, M.C. Innovation management: a literature review about the evolution and the different 
innovation models. XVIII International Conference on Industrial Engineering and Operations Management. Guimaraes - Portugal: ABEPRO: 8 p. 2012.

MACIAS-CHAPULA, C.A. O papel da informetria e da cienciometria e sua perspectiva nacional e internacional. Ciência da informação, v. 27, n. 2, p. 134-140, 1998.

http://dx.doi.org/10.1590/S0100-19651998000200005

MEHO, L.I.; YANG, K. Impact of data sources on citation counts and rankings of LIS faculty: Web of Science versus Scopus and Google Scholar. Journal of the american society for information science and technology, v. 58, n. 13, p. 2105-2125, 2007.

http://dx.doi.org/10.1002/asi.20677

MGH. Massachusetts General Hospital - official web site. 2014. Disponível em: < http://www.massgeneral.org/ >. Acesso em: 10 Jan 2015.

MINISTÉRIO DA SAÚDE. Portaria n 4.279, de 30 de Dezembro de 2010. 2010.

Disponível em: <

http://bvsms.saude.gov.br/bvs/saudelegis/gm/2010/prt4279 3012 2010.html >. Acesso em: 28 Jul 2014.

NG, D.; VAIL, G.; THOMAS, S.; SCHMIDT, N. Applying the Lean principles of the Toyota Production System to reduce wait times in the emergency department. Canadian Journal of Emergency Medicine, v. 12, n. 1, p. 50-57, Jan 2010.

OHNO, T. O Sistema Toyota de Produção: além da produção em larga escala. Artmed, 1997. 152p.

PINTO, L.R.; CAMPOS, F.C.C.D.; PERPÉTUO, I.H.O.; RIBEIRO, Y.C.N.M.B. Analysis of Hospital Bed Capacity via Queuing Theory and Simulation. 2014 Winter Simulation Conference. Savannah, GA 2014.

RUTLEDGE, J.; XU, M.; SIMPSON, J. Application of the Toyota Production System improves core laboratory operations. American Journal of Clinical Pathology, v. 133, n. 1, p. 24-31, 2010. http://dx.doi.org/10.1309/AJCPD1MSTIVZIOPZ

SERAPHIM, E.C.; DA SILVA, I.; AGOSTINHO, O.L. Lean Office em organizações militares de saúde: estudo de caso do Posto Médico da Guarnição Militar de Campinas. Gestão \&. Produção, p. 389-405, 2010. http://dx.doi.org/10.1590/S0104-530X2010000200013

SHINGO, S. Sistema Toyota de Producao do ponto de vista da Engenhria de Produção. Bookman, 1996. 292p.

SILVA, E.N.; SANTOS, G.T. A aplicação dos conceitos do sistema Toyota de produção em uma empresa prestadora de serviços. Encontro Nacional de Engenharia de Produção. Salvador: ABEPRO 2001.

SOUSA, D.G.; SILVEIRA, L.F.; BAGNO, R.B. Aplicabilidade dos princípios do Lean Manufacturing no setor de serviços: estudo em uma oficina mecânica de motos. $X X X I I$ Encontro Nacional de Engenharia de Produção. Bento Gonçalves, RS: ABEPRO 2012.

USDVA. Veterans Health Administration. 2014. Disponível em: < http://www.va.gov/health/ >. Acesso em: 10 Jan 2014. 
VALLE, L.D.A.; GUTIERREZ, R.H.; COSTA, H.G. Estudo Bibliométrico da Produção Científica em Gestão de Processos na Saúde no Brasil. XXXIII Encontro Nacional de Engenharia de Produção. Salvador: ABEPRO 2013.

WSC. Proceedings of the $\mathbf{2 0 1 4}$ Winter Simulation Conference. In: BUCKLEY, S. J. e MILLER, J. A., Winter Simulation Conference, 2014. Savannah, GA. IEEE Press.

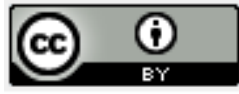

Artigo recebido em 24/07/2015 e aceito para publicação em 01/10/2015

DOI: http://dx.doi.org/ 10.14488/1676-1901.v16i1.2088 